\title{
Publisher Correction: Heat stress activates YAP/TAZ to induce the heat shock transcriptome
}

Min Luo (iD, Zhipeng Meng, Toshiro Moroishi (iD, Kimberly C. Lin, Guobo Shen (D), Fei Mo, Bin Shao, Xiawei Wei (iD, Ping Zhang, Yuquan Wei and Kun-Liang Guan (D)

Correction to: Nature Cell Biology https://doi.org/10.1038/s41556-020-00602-9, published online 16 November 2020

In the version of this Article originally published, there were thick black lines covering values in the lower middle graph of Extended Data Fig. 5g. The error has been corrected.

Published online: 11 December 2020

https://doi.org/10.1038/s41556-020-00623-4

(c) The Author(s), under exclusive licence to Springer Nature Limited 2020 